\title{
Relation of Forbush decrease with interplanetary magnetic fields.
}

\author{
K. P. Arunbabu ${ }^{* a, b}$, H. M. Antia ${ }^{a, b}$, S. R. Dugad ${ }^{a, b}$, S. K. Gupta ${ }^{a, b}$, Y. Hayashi ${ }^{a, d}$, S. \\ Kawakami $^{a, d}$, P. K. Mohanty ${ }^{a, b}$, A. Oshima ${ }^{a, e}$, P. Subramanian ${ }^{a, c}$ \\ ${ }^{a}$ The GRAPES-3 Experiment, Cosmic Ray Laboratory, Raj Bhavan, Ooty 643 001, India \\ ${ }^{b}$ Tata Institute of Fundamental Research, Homi Bhabha Road, Mumbai 400 005, India \\ ${ }^{c}$ Indian Institute of Science Education and Research, Dr. Homi Bhabha Road, Pune 411 021, \\ India \\ ${ }^{d}$ Graduate School of Science, Osaka City University, Osaka 558-8585, Japan \\ ${ }^{e}$ College of Engineering, Chubu University, Kasugai, Aichi 487-8501, Japan \\ E-mail: arun.babuetifr.res.in
}

\begin{abstract}
The relation between the Forbush decreases (FDs) and near-Earth interplanetary magnetic field (IMF) enhancements associated with the solar coronal mass ejections (CMEs) is studied. We have used data from GRAPES-3 tracking muon telescope to identify the Forbush decrease events. We have chosen events that are having a reasonably clean profile, and magnitude $>0.25 \%$. We have used IMF data from ACE/WIND spacecrafts to investigate how closely the FD profile follow the IMF enhancements. We found that the enhancement of magnetic field responsible for the FD takes place mainly in the sheath region and also the MHD turbulence level get enhanced in this region. We found that the FD profile looks remarkably similar to that of IMF enhancement, yielding good correlation with a time lag. The FD profile lags behind the IMF by few hours. This observed lag corresponds to the time taken by high energy protons to diffuse into the magnetic field enhancement through cross-field diffusion.
\end{abstract}

The 34th International Cosmic Ray Conference,

30 July- 6 August, 2015

The Hague, The Netherlands

\footnotetext{
* Speaker.
} 


\section{Introduction}

Forbush decreases (FDs) are transient decreases in the observed galactic cosmic ray intensity, which were first reported by Forbush [1,2]. Their connection to interplanetary magnetic field was first reported by Simpson [3]. Solar activities such as coronal mass ejections (CMEs) cause disturbances in the interplanetary magnetic field (IMF). Some CMEs ejected from Sun travel in the Sun-Earth line and can cause disturbances in the near Earth IMF. There are two major component of these CMEs, i) the interplanetary counterpart of CME, commonly known as ICME and ii) the shock propagating ahead of ICME. Both shock and ICME can cause significant enhancement in the IMF. Correlation between the parameters characterizing FD and solar wind parameters have been a subject of considerable study $[4,5,6]$.

\section{Data analysis}

We used data from GRAPES-3 experiment located at Ooty $\left(11.4^{\circ} \mathrm{N}\right.$ latitude, $76.7^{\circ} \mathrm{E}$ longitude, and $2200 \mathrm{~m}$ altitude) in India. This experiment contains two major components, first an air shower array of 400 scintillation detectors (each of $1 \mathrm{~m}^{2}$ ) arranged in a hexagonal geometry, with a distance of $8 \mathrm{~m}$ between adjacent detectors $[7,8,9,10]$. The second component of GRAPES-3 experiment is a large area tracking muon telescope. This muon telescope is a unique instrument used to search for high energy protons emitted during the active phase of a solar flare or a CME, which provides a high statistics, directional measurement of the muon flux. The GRAPES-3 muon telescope covers an area of $560 \mathrm{~m}^{2}$, consisting of a total of 16 modules, each $35 \mathrm{~m}^{2}$ in area. The energy threshold of the telescope is $\sec (\theta) \mathrm{GeV}$, for the muons arriving along a direction with zenith angle $\theta$. The observed muon rate of $\sim 3000 \mathrm{~s}^{-1}$ per module, yields a total muon rate $\sim 3 \times 10^{6} \mathrm{~min}^{-1}$ for the entire telescope [11, 12]. This large rate permits even a small change of $\lesssim 0.1 \%$ in the muon flux to be measured accurately over a time scale of $\sim 5 \mathrm{~min}$, after appropriate corrections are applied for the time dependent variation in the atmospheric pressure [13].

The Grapes-3 muontracking telescope has a capability of getting data from 169 directions. These directions were regrouped in a suitable manner to form nine different directions, named NW (northwest), N (north), NE (northeast), W (west), V (vertical), E (east), SW (southwest), S (south), and SE (southeast) with uniform solid angle in field of view [12]. The cutoff rigidity due to the geomagnetic field at Ooty along the vertical direction is $17 \mathrm{GV}$ and it varies from 14 to $42 \mathrm{GV}$ across the 2.2 sr. field of view of the muon telescope. Details of the muon telescope are given in $[11,12,14]$.

The GRAPES-3 muon telescope has observed large number of FDs. In this study we analyzed FD events during year 2001 to 2004, out of which which we chose FD events having a clean Forbush decrease profile (sudden decrease and gradual recovery in cosmic ray flux), magnitude $>0.25 \%$, and associated with a near-Earth enhancement in the IMF. Although $0.25 \%$ might seem like a small number, considering the high sensitivity of this instrument these are fairly significant events [15]. We used GRAPES-3 data summed over a time interval of one hour for each directions, which improved the signal-to-noise ratio. Diurnal anisotropy is present in this data [13]. We used a low pass filter to remove these diurnal anisotropy, which removes all the frequencies $>d a y^{-1}$ [14]. 
FD we studied are associated with IMF compressions due to near-Earth CMEs. We used the IMF data observed by ACE and WIND spacecrafts available from OMNI database. We used hourly resolution data on magnetic field $\mathrm{B}_{\text {total }}, \mathrm{B}_{\mathrm{x}}, \mathrm{B}_{\mathrm{y}}, \mathrm{B}_{\mathrm{z}}$ in the geocentric solar ecliptic (GSE) coordinate system: $\mathrm{B}_{\text {total }}$ is the magnitude of the magnetic field, $\mathrm{B}_{\mathrm{x}}$ is the magnetic field component along the Sun-Earth line in the ecliptic plane pointing towards the Sun, $B_{z}$ the component parallel to the ecliptic north pole, and $\mathrm{B}_{\mathrm{y}}$ the component in the ecliptic plane pointing towards dusk. Since enhancement in IMF are associated with FD, we used the quantity $100-|\mathrm{B}|$ and calculate the average value and the percent deviation of this quantity over the same data interval as the FD. This effectively flips IMF and make the enhancement appear like a decrease, enabling easy comparison with the FD profile. Figure 1 shows an example of a FD event observed on 23 May 2002, together with the IMF data processed in this manner.

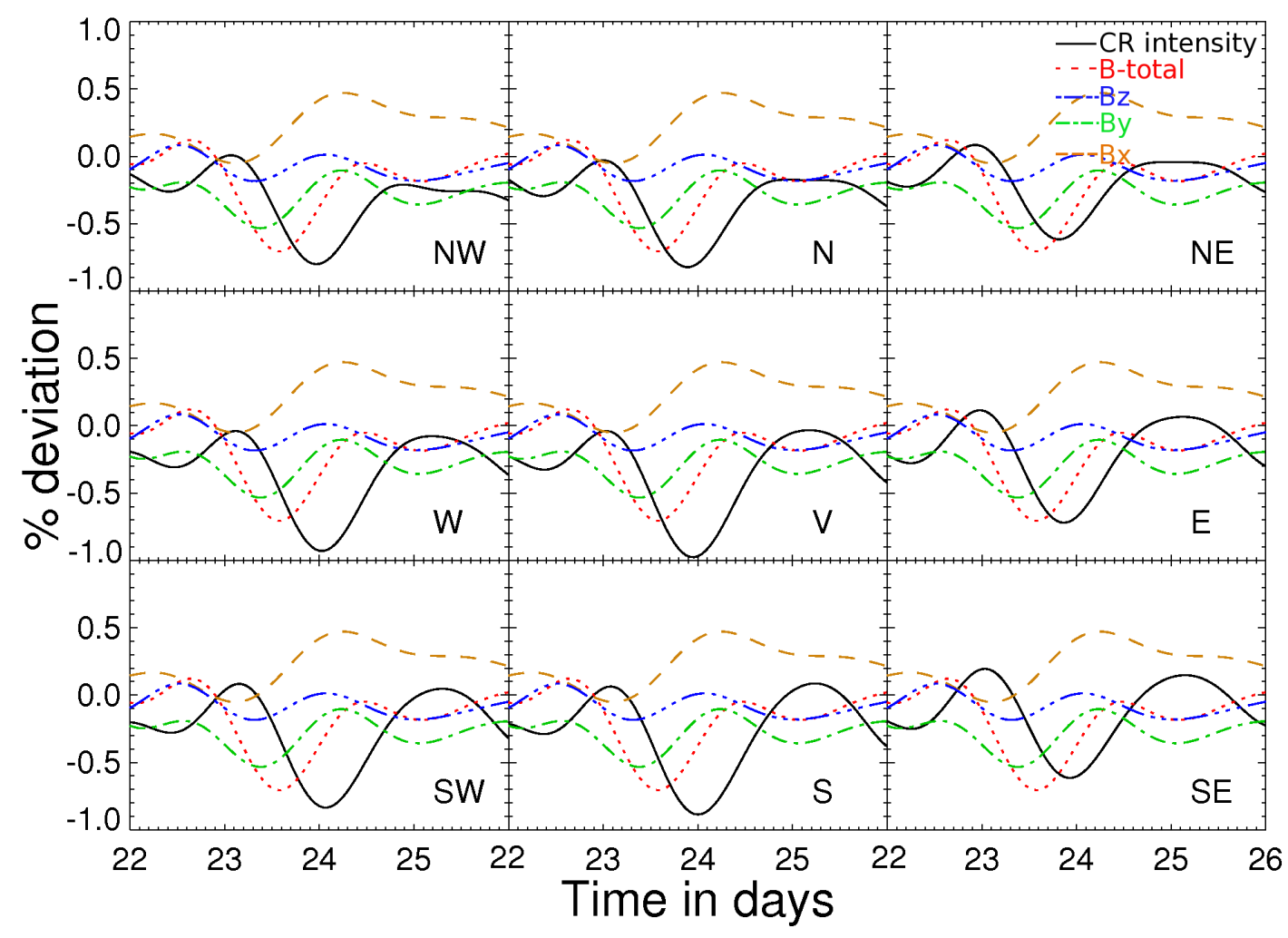

Figure 1: The FD event of 23 May 2002 and the magnetic field for 9 directions in GRAPES-3 muon telescope. Black solid line is percentage deviation of cosmic ray intensity in each direction. The red-dotted, blue-dash-dot-dotted, green-dash-dotted and orange-dash lines are percentage deviation of IMFs $\mathrm{B}_{\text {total }}, \mathrm{B}_{\mathrm{z}}$, $\mathrm{B}_{\mathrm{y}}$ and $\mathrm{B}_{\mathrm{x}}$ respectively, that are scaled down by a factor of 10 to fit in the frame.

\section{Correlation FD magnitude with peak IMF}

We first studied the correlation of FD magnitude with the maximum magnetic field in the magnetic field compression. We studied 65 FD events chosen with the criteria mentioned in section 2. The FD magnitude is calculated as difference between the pre-event cosmic ray intensity to the 
intensity at the minimum of decrease. We examined the corresponding IMF during these events. Since for an ideal fluxrope CME approaching Earth, the $B_{x}$ component of IMF will be the radial magnetic field of fluxrope and $B_{y}$ and $B_{z}$ will be perpendicular components as appear to a typical cosmic ray particle trying to diffuse in to the fluxrope CME. So in this study we consider two derived IMF components the scalar magnetic field $\left(\mathrm{B}_{\text {total }}=\left(\mathrm{B}_{\mathrm{x}}^{2}+\mathrm{B}_{\mathrm{y}}^{2}+\mathrm{B}_{\mathrm{z}}^{2}\right)^{1 / 2}\right.$ and the perpendicular magnetic field $\left(\mathrm{B}_{\mathrm{p}}=\left(\mathrm{B}_{\mathrm{y}}^{2}+\mathrm{B}_{\mathrm{z}}^{2}\right)^{1 / 2}\right)$. The correlation coefficients of peak $B_{\text {total }}$ and $B_{p}$ are listed in the table 1 .

Table 1: Correlation of the FD magnitude with the maximum total and perpendicular IMF. For each direction of GRAPES-3, the correlation is calculated and are shown along with Cut-off rigidity.

\begin{tabular}{lccccccccc}
\hline \hline Direction & NW & N & NE & W & V & E & SW & S & SE \\
Cut-off Rigidity (GV) & 15.5 & 18.7 & 24.0 & 14.3 & 17.2 & 22.4 & 14.4 & 17.6 & 22.4 \\
$B_{\text {total }}$ Corr. coeff. & 0.702 & 0.707 & 0.720 & 0.684 & 0.688 & 0.681 & 0.669 & 0.660 & 0.636 \\
$B_{p}$ Corr. coeff. & 0.712 & 0.714 & 0.724 & 0.691 & 0.692 & 0.685 & 0.676 & 0.666 & 0.642 \\
\hline \hline
\end{tabular}

The FD magnitude depends on various parameters associated with CME other than magnetic field, such as velocity of CME, turbulence level in magnetic field, and size of CME [15]. Thus it is not surprising that the FD magnitude correlates only moderately with the peak value of CME.

\section{IMF compression}

We considered a subset of events which are having a well-defined shock and ICME/magnetic cloud associated with the corresponding magnetic field compression. An example of such an event is shown in figure 2, in which FD profile for 9 different directions are shown along with magnetic field. The vertical lines corresponds to timing of shock arrival, magnetic cloud start, magnetic cloud end, maximum magnetic field compression, FD onset, and FD minimum. It is clear from the figure that the magnetic field compression responsible for the FD is in the sheath region (region between the shock and ICME).

The cross field diffusion of cosmic rays through turbulent magnetic field depends on rigidity of proton and the turbulence level in magnetic field $(\sigma)$ [16], hence it is important to study the turbulence level in this magnetic field compressions. We have calculated the turbulence level using one-minute averaged data from the ACE/WIND spacecraft. Turbulence level $\sigma$ has been calculated using a one-hour running average of the magnetic field $\left(\mathrm{B}_{0}\right)$ and the fluctuation of the IMF around this average $\left(\mathrm{B}_{\text {tur }}=\mathrm{B}-\mathrm{B}_{0}\right)$. We define the quantity $\sigma$ as

$$
\sigma=\left(\frac{\left\langle\mathrm{B}_{\mathrm{tur}}^{2}\right\rangle}{\mathrm{B}_{0}^{2}}\right)^{0.5}
$$

where $\left\langle\mathrm{B}_{\text {tur }}^{2}\right\rangle$ denotes the average of $\mathrm{B}_{\text {tur }}^{2}$ over the one-hour window. Figure 3 shows a representative event.

We note that the magnetic field enhancement takes place in the sheath region, i, e., the region between shock and CME, the magnetic turbulence level, $\sigma$ is also get enhanced in this region. According to the CME-only cumulative diffusion model high energy cosmic rays are diffusing in to the CME through this turbulent magnetic field region [15]. 


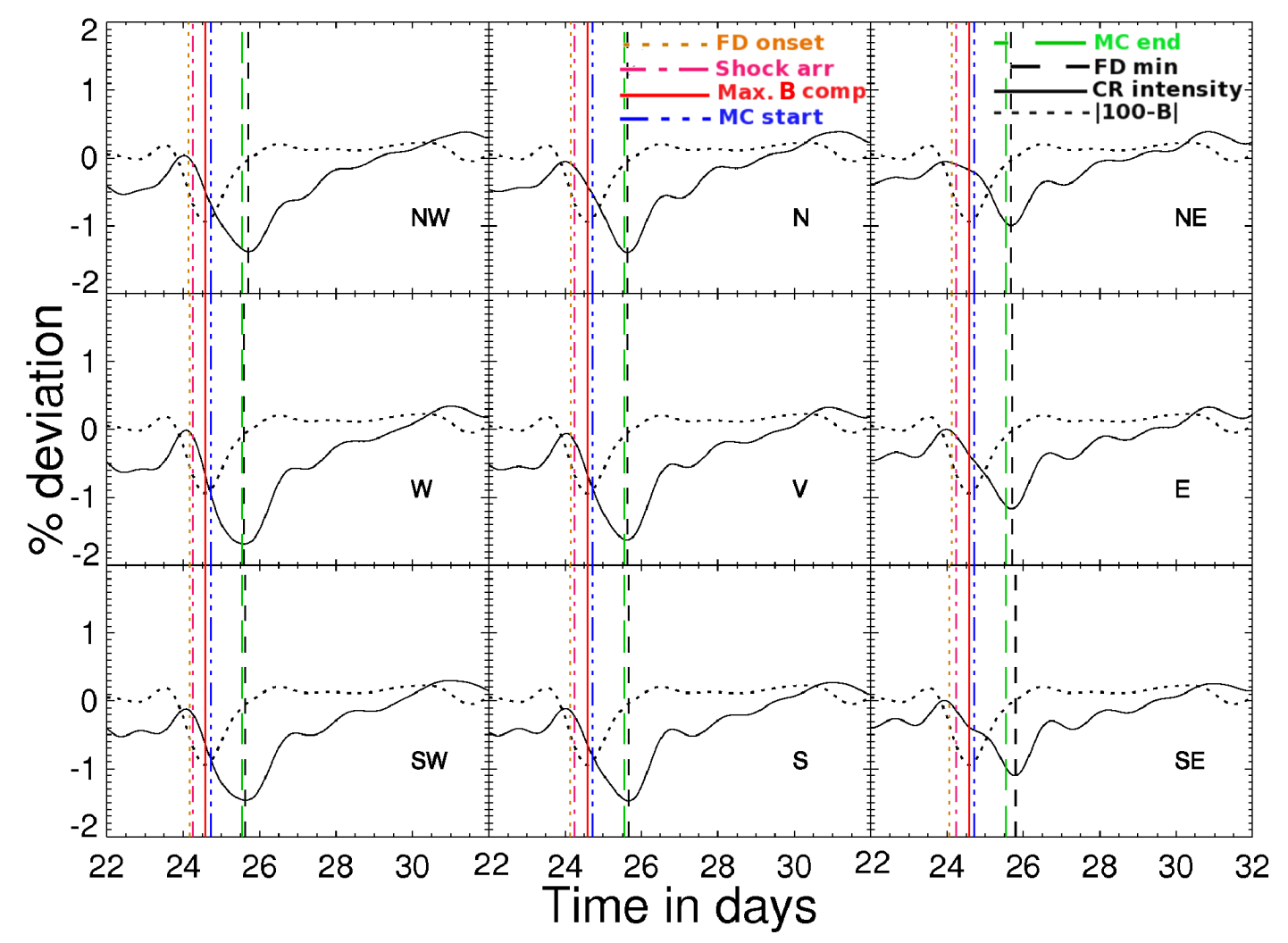

Figure 2: FD event on 24 November 2001. The black solid line denotes percentage deviation of the cosmic ray intensity, black dotted line percentage deviation of total magnetic field $|100-\mathrm{B}|$ which is scaled down to fit the frame. The vertical brown-dotted, magenta-dash-dotted, blue-dash-dot-dotted, green-long-dashed, and black-dashed lines denote the timings corresponding to the FD onset, shock arrival, magnetic cloud start, magnetic cloud end, and FD minimum, respectively. The solid red vertical line corresponds to the maximum of the magnetic field compression.

\section{Comparing FD and IMF profiles}

Near-Earth CMEs cause a magnetic field compression in the IMF. We investigated the relation of this magnetic field compression to the FD profile for each individual events separately. For this study we took the IMF hourly resolution IMF data from ACE/WIND spacecrafts. A visual comparison of FD profile with the IMF compression always shows a remarkable similarity. To quantify this we studied the cross-correlation of cosmic ray intensity profile with the IMF compression profile. In order to do this we shift the magnetic field profile with respect to FD profile by amounts of -36 to 12 hours. We identify the peak correlation value and the shift corresponding to this value as the lag between the IMF and FD profiles. Cross correlation of 23 May 2002 event is shown in figure 4 as an example, which shows a high correlation of $98 \%$ with a lag of $13 \mathrm{hrs}$. We have carried out this for all the events we shortlisted most of most of the events show correlation $>60 \%$. Events which show good correlation with the perpendicular magnetic fields are listed in table 2. 

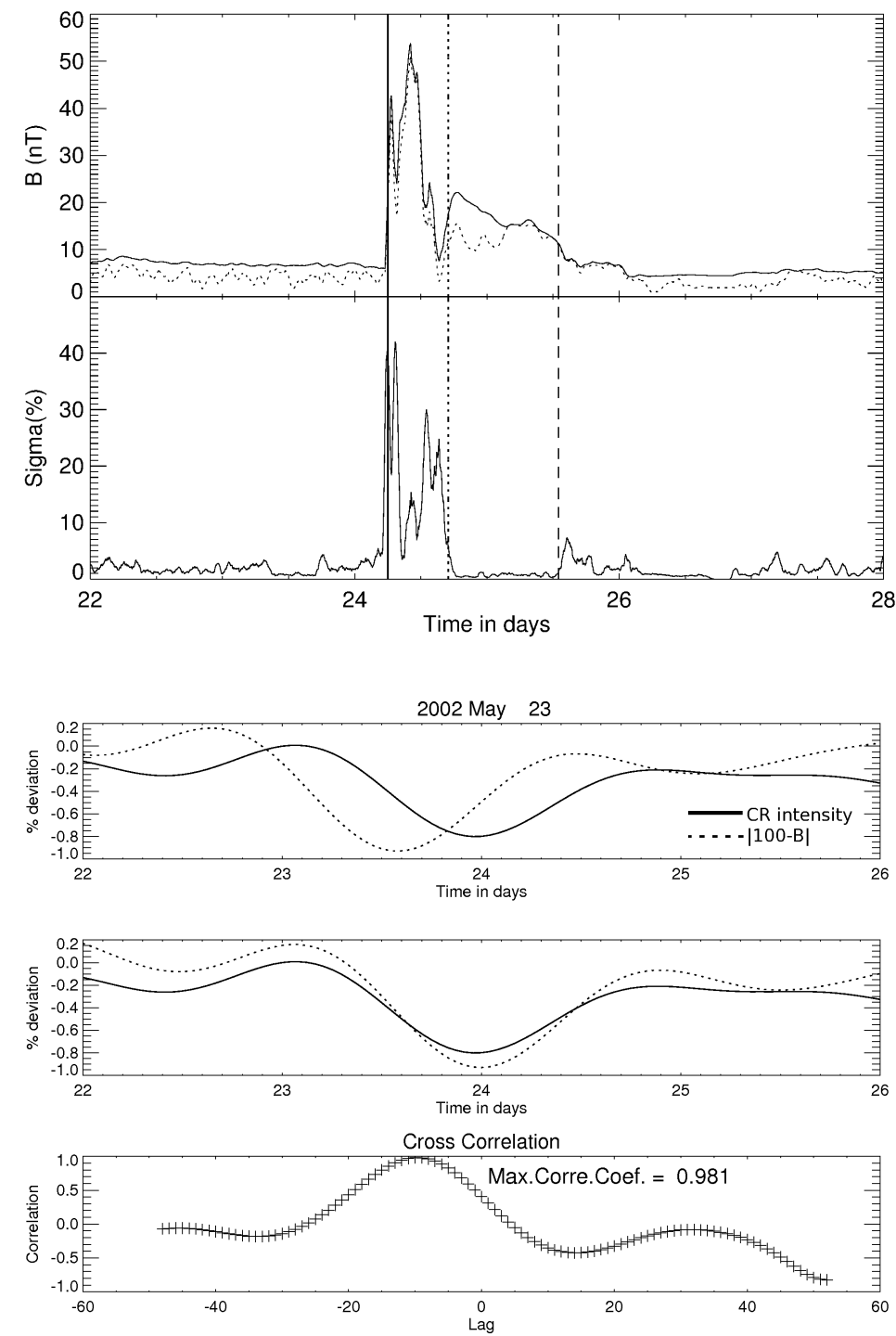

Figure 3: Magnetic field compression associated with the FD event on 24 November 2001. In the first panel the continuous line denotes $B_{\text {total }}$ and the dotted line denotes $B_{p}$. The continuous line in the second panel shows the turbulence level for $\mathrm{B}_{\text {total }}$. In both panels the vertical solid, dotted, and dashed lines corresponds to shock arrival time, magnetic cloud start time, magnetic cloud end time, respectively.

Figure 4: Cross correlation of the cosmic ray flux with $\mathrm{B}_{\text {total }}$. The top panel shows the percentage deviation of cosmic ray flux using solid black lines and the magnetic field using dotted black lines (scaled to fit in the frame). The middle panel shows the same with magnetic field shifted to the right corresponding to the time lag and the bottom panel shows the correlation coefficient for different lags.

\section{IMF compression-FD profile lag: cosmic ray diffusion}

The observed lag between the cosmic ray flux and IMF is poorly correlated with the FD magnitude and CME speed. This lag occurs because the high-energy protons are not responding to the IMF compression instantaneously; they are subjected to the classical magnetic mirror effect arising from the gradient in the longitudinal magnetic field and to turbulent cross-field (also referred as perpendicular) diffusion [17]. In this study we are concentrating on the cross-field diffusion of high energy particles through the turbulent sheath region. The perpendicular diffusion coefficient depends on the rigidity of the particle and the magnetic turbulence level $\sigma$ [16].

We interpret the observed time lag between the IMF and the FD profiles as the time taken by the high-energy protons to diffuse through the turbulent magnetic field compression. The time taken for a single diffusion random walk of a high-energy proton into the magnetic structure of CME is given by 
Table 2: Events for which the FD profile correlates well only with the perpendicular component of the IMF enhancement.

\begin{tabular}{|c|c|c|c|c|c|c|c|c|c|}
\hline \multirow[t]{2}{*}{ Event } & \multicolumn{3}{|c|}{$\mathrm{B}_{\text {total }}$} & \multicolumn{3}{|c|}{$\mathrm{B}_{\mathrm{y}}$} & \multicolumn{3}{|c|}{$\mathrm{B}_{\mathrm{z}}$} \\
\hline & $\begin{array}{l}\text { Corr. } \\
(\%)\end{array}$ & $\begin{array}{l}\text { Lag } \\
(\mathrm{hrs})\end{array}$ & $\begin{array}{c}\text { No of } \\
\text { diff. }\end{array}$ & $\begin{array}{c}\text { Corr. } \\
(\%)\end{array}$ & $\begin{array}{c}\text { Lag } \\
(\mathrm{hrs})\end{array}$ & $\begin{array}{c}\text { No of } \\
\text { diff. }\end{array}$ & $\begin{array}{c}\text { Corr. } \\
(\%)\end{array}$ & $\begin{array}{l}\mathrm{Lag} \\
\text { (hrs) }\end{array}$ & $\begin{array}{c}\text { No of } \\
\text { diff. }\end{array}$ \\
\hline 2001 Jan 13 & 97.2 & -13 & 384 & 95.8 & -14 & 196 & 96.6 & -23 & 97 \\
\hline 2001 Apr 11 & 90.7 & -19 & 656 & 89.9 & -17 & 326 & 91.3 & -5 & 41 \\
\hline 2001 May 27 & 66.5 & 0 & 0 & 65.0 & -3 & 44 & 75.7 & -21 & 111 \\
\hline 2001 Aug 13 & 51.8 & -7 & 216 & 97.5 & -7 & 76 & 70.0 & -5 & 26 \\
\hline 2001 Sep 12 & 79.2 & -25 & 838 & 26.7 & -30 & - & 86.2 & -1 & 17 \\
\hline 2001 Nov 24 & 85.3 & -21 & 822 & 41.0 & -31 & 173 & 77.1 & -14 & 260 \\
\hline 2001 Dec 14 & 74.7 & -35 & 1346 & 69.7 & -2 & 56 & 72.8 & -17 & 255 \\
\hline 2002 Sep 07 & 77.1 & -18 & 776 & 49.6 & -24 & 312 & 87.4 & 3 & 24 \\
\hline 2002 Sep 30 & 81.1 & -5 & 207 & 72.1 & 8 & 130 & 75.7 & -12 & 198 \\
\hline 2002 Dec 22 & 73.4 & -15 & 481 & 43.4 & 0 & 0 & 84.7 & -12 & 66 \\
\hline 2003 Jan 23 & 70.9 & -21 & 509 & - & - & - & 75.4 & -28 & 49 \\
\hline 2003 Feb 16 & - & - & - & - & - & - & 74.3 & -11 & 55 \\
\hline 2003 May 04 & 83.4 & -8 & 252 & 84.7 & -10 & 97 & 80.7 & 0 & 0 \\
\hline 2003 Jul 25 & 95.3 & -19 & 565 & 73.3 & -2 & 32 & 41.6 & 2 & 9 \\
\hline 2003 Dec 27 & 86.1 & -35 & 1210 & 21.7 & 5 & 41 & 87.2 & -3 & 10 \\
\hline 2004 Aug 30 & - & - & - & - & - & - & 92.4 & 1 & 10 \\
\hline 2004 Dec 05 & 85.3 & -12 & 326 & 89.4 & 8 & 107 & 58.4 & -13 & 117 \\
\hline 2004 Dec 12 & 81.1 & -17 & 595 & 73.3 & -25 & 243 & 78.9 & -13 & 138 \\
\hline
\end{tabular}

$$
t_{d i f f}=\frac{D_{\perp}}{c V_{s w}},
$$

where $D_{\perp}$ is the perpendicular diffusion coefficient [16], c is the speed of light (which is the typical propagation speed for the highly relativistic galactic cosmic rays we are concerned with) and $\mathrm{V}_{\mathrm{sw}}$ is the solar wind velocity upstream of the CME.

We estimated the number of diffusion lengths required to account for the observed time lag between the IMF and FD profile using,

$$
\text { No. of Diffusions }=\frac{\text { Lag }}{\mathrm{t}_{\text {diff }}}
$$

This number of diffusion is calculated using the peak value of IMF compression. It is evident that the observed lag can be accounted for by a few tens to a few hundred diffusion times and is shown in table 2.

\section{Conclusions}

Our previous study [15] show that FDs are due to cumulative diffusion of galactic cosmic ray protons into the CME as it propagates from the Sun to the Earth. However, the precise nature of the 
diffusive barrier was left unspecified, and the diffusion was assumed to occur across an idealized thin boundary that presumably had to do with the turbulent sheath region. The results from this work show that the magnetic field enhancement responsible for the FD comprises the sheath region. The FD profile looks like a lagged (and inverted) copy of the enhanced magnetic field compression (Figure $4 \&$ Table 2). The FD lags behind the magnetic field enhancement by a few hours (Table 2). We have quantitatively shown that the observed time lag between the FD and IMF enhancement can be accounted for by cross-field diffusion through the turbulent sheath region (Table 2). This work establishes

- i) the importance of the turbulent sheath region between the shock and ICME; we showed that the magnetic field enhancement responsible for the FD comprises the shock-sheath, and the magnetic turbulence level is also enhanced in this region.

- ii) the viability of cross-field diffusion through the turbulent CME sheath as the primary mechanism for FDs .

\section{References}

[1] Forbush, S.E., 1937, Phys. Rev. 51, 1108

[2] Forbush, S. E., 1938, Phys. Rev, 54, 975.

[3] Simpson, J. A., 1954, Phys. Rev., 94, 426

[4] Belov, et. al., 2001, AdSpR, 27, 625B

[5] Kane, R. P., 2010, AnGeo, 28, 479

[6] Dumbović, M., et. al., 2012, A\&A, 538, 28

[7] Gupta, S. K., et al., 2005, Nucl. Instrum. Methods A, 540, 311

[8] Gupta, S. K., et al., 2009, Nucl. Phys. B Proc. Suppl., 196, 153

[9] Mohanty, P. K., et al., 2009, Astropart. Phys. 31, 24

[10] Mohanty, P. K., Dugad, S. R., \& Gupta, S. K., 2012, Rev. Sci. Instrum. 83, 043301

[11] Hayashi, Y., et al., 2005, Nucl. Instrum. Methods A, 545, 643

[12] Nonaka, T., et al., 2006, Phys. Rev. D., 74, 052003

[13] Mohanty, P. K., et al., 2013, Pramana J. Phys., 81, 343

[14] Subramanian, P., et. al., 2009, A\&A, 494, 1107

[15] Arunbabu, K. P., et. al., 2013, A\&A, 555A, 139A

[16] Candia, J., \& Roulet, E. 2004, J. Cosmology \& Astropart. Phys., 10, 007

[17] Kubo, Y., \& Shimazu, H., 2010, ApJ, 720, 853 